\title{
Large mucocele of appendix
}

\author{
Palaniswami Kamalesh • Kurumboor Prakash • \\ Kaniyarakal Pramil • I. S. Vipin
}

Published online: 2 May 2012

(C) Indian Society of Gastroenterology 2012

A 50-year-old lady presented with right-sided abdomen pain of three days duration. She had no other complaints. Her clinical assessment, hemogram, liver function tests and serum tumor markers were within normal limits. CT scan of abdomen showed a $15 \mathrm{~cm} \times 5 \mathrm{~cm} \times 5 \mathrm{~cm}$ elongated, fluid-filled, thick-walled structure, with curvilinear calcification contiguous with the base of the cecum, and extending into the pelvis on the right side of abdomen (Fig. 1). A preoperative diagnosis of appendiceal mucocele was made. Colonoscopy showed an extrinsic bulge in the ascending colon. Laparoscopy confirmed an appendiceal mucocele measuring $17 \mathrm{~cm} \times 6 \mathrm{~cm}$ in size. She underwent laparosocopic appendicectomy. The appendix contained thick, viscid mucus, and histopathology confirmed it as a mucinous cystadenoma.

Appendiceal mucocoele is characterized by cystic dilation of the lumen due to obstruction and consequent accumulation of mucus. The incidence is $0.2-0.3 \%$ and is more common in elderly women. Only $50 \%$ of patients are symptomatic; in the rest it is incidentally detected during surgery [1]. A preoperative diagnosis is rarely made; CT scan and ultrasonogram are valuable in establishing the diagnosis. At CT scan, mucocoele appears as a well-encapsulated, round, thin-walled cystic mass. Presence of curvilinear or punctuate wall calcification strongly suggests the diagnosis [2]. All these CT characteristics were seen in our patient as well.

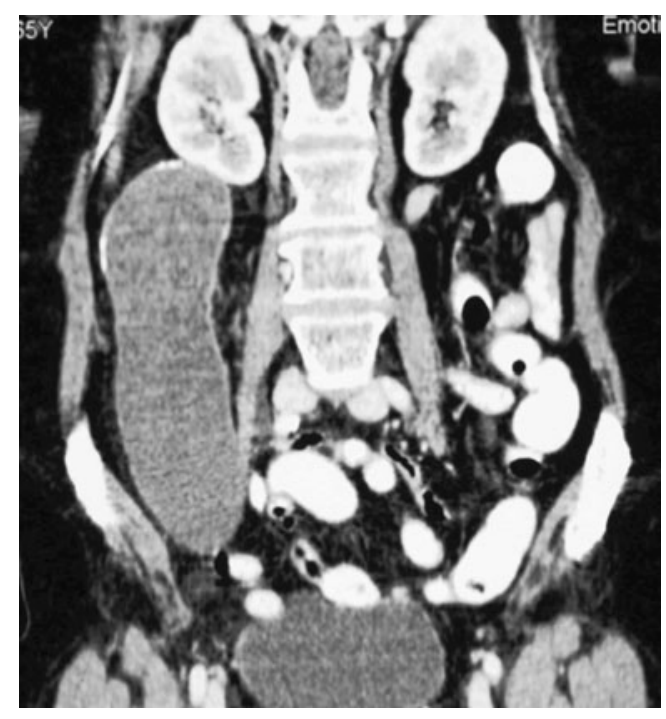

Fig. 1 CT scan shows large mucocele with calcification

\section{References}

1. Dixit A, Robertson JH, Mudan SS, Akle C. Appendiceal mucocoeles and pseudomyxoma peritonei. World J Gastroenterol. 2007;13:2381-4.

2. Madwed D, Mindelzun R, Jeffrey Jr RB. Mucocele of the appendix: imaging findings. AJR Am J Roentgenol. 1992;159:69-72.

P. Kamalesh $(\bowtie) \cdot K$. Prakash $\cdot$ K. Pramil • I. S. Vipin

Department of Gastrointestinal Surgery,

P V S Institute of Digestive Diseases,

Cochin, Kerala 682 017, India

e-mail:kamli6@yahoo.co.uk 